\title{
Adult-onset epidermal nevus with epidermolytic hyperkeratotic pattern: Case report, dermoscopic features, and review of the literature.
}

\author{
Mahshid Sadat Ansari ${ }^{1}$, Maryam Nasimi ${ }^{1}$, Kambiz Kamyab ${ }^{1}$, and Hamidreza Mahmoudi ${ }^{1}$ \\ ${ }^{1}$ Tehran University of Medical Sciences
}

May 11, 2020

\begin{abstract}
Epidermolytic hyperkeratotic EN (epidermal nevus) is a rare variant of EN, which can be congenital or developed later in adulthood. A 23 years old female presented with a pathologic confirmed epidermolytic hyperkeratotic EN. Dermoscopy can be helpful in the diagnosis of EN, but not in differentiating the variant.
\end{abstract}

\section{Introduction}

Epidermal nevus (EN) is a hamartomatous proliferation of keratinocytes. It usually appears during the first year of life and gradually increases in thickness, although some cases of late-onset development have also been reported ${ }^{1}$. EN is usually an isplated benign lesion, but especially when it is extensive, it may be associated with other organ and system abnormalities which is called epidermal nevus syndrome ${ }^{2}$.

Dermoscopy is a non-invasive diagnostic method that can be used readily to differentiate melanocytic and non-melanocytic lesions. It also helps in biopsy decision making for a suspected lesion ${ }^{2 / 3}$.

Herein we report an acquired case of EN in a 32-year-old woman with epidermolytic hyperkeratotic pattern and present a review of the literature about it.

\section{Case presentation}

A 32-year-old woman presented with a unilateral small brown blaschkoid sessile and pedunculated papules on the right side of her abdomen in the past 4 years. She did not complain from any symptom except for occasional pruritus. The lesions appeared during her first pregnancy and became more prominent and increased in number during her second pregnancy. Past medical history and examination were otherwise unremarkable.

On dermoscopic examination multiple round and oval brown pigmented circles with irregular borders and some smaller circles were seen in a homogenous light brown background(Fig 1).

Histology examination revealed a thick orthokeratosis, papillomatosis, coarse keratohyalin granules, and some vacuolated cells with dark basophilic nuclei known as epidermolytic hyperkeratotic pattern. Some superficial lymphocytic infiltration was also noted in the dermis. Regarding the history, clinical manifestation and histopathologic findings diagnosis of epidermolytic hyperkeratotic EN was made (Fig 2). 


\section{Disscusion}

EN is fundamentally a hamartoma of keratinocytes and occasionally adnexal structures. It mostly develops in the first years of life, although late-onset presentation has also been reported ${ }^{1 '} 4$. Martinez et al report a 27 years old woman who developed EN during pregnancy. They proposed hormonal and immunological alternations during pregnancy as a possible explanation 5 .

More than 10 histopathological variants of EN have been described so far and papillomatosis, hyperkeratosis, and acanthosis are the most common findings. Epidermolytic hyperkeratosis is an uncommon variant 6 .

Epidermolytic hyperkeratosis is characterized by granular and vacuolar degeneration of keratinocytes in the spinous and granular layer of epidermis along with hyperkeratosis. In addition to EN, this histological pattern can be seen in epidermolytic ichthyosis, specific kinds of palmoplantar keratodermas and epidermolytic acanthoma 7 .

The dermoscopy view of the epidermolytic hyperkeratosis EN is mostly similar to other kinds of EN containing: large brown circles and the absence of the pigment network ${ }^{2}$ '8. Carbotti et al first described large brown circles in EN and correlated it histologically to the way pigmented keratinocytes lie down around dermal papillae ${ }^{2}$. Demographics and dermoscopic features of epidermolytic hyperkeratosis EN being reported in the literature are summarized in Table 1.

The clinical differential diagnosis of this lesion includes wart and seborrheic keratosis which can be excluded by considering the unique clinical pattern of EN (blaschkoid distribution) and lack of cytopathic viral effects, which are seen in the wart.

Full-thickness surgical excision is the treatment, but in large lesions hypertrophic scar and keloid formation is the side effect. Other treatment modalities include electrofulguration, cryosurgery, and dermabrasion, but they make scarring. Topical agents (calcipotriol, steroids, retinoic acid) were not effective. Soft and flat nevi were responsive to ablative lasers, but the lesions may recur after non-surgical procedures. So because of the side effects of extensive surgery a conservative approach is logic in the case of extensive lesions ${ }^{9}$.

In conclusion epidermolytic hyperkeratotic EN is a rare variant of EN, which can be congenital or developed later in adulthood. Although the histopathological finding of this variant is unique and is different from other variants of EN, the dermoscopic feature is the same and can be considered an important clue in the diagnosis of different variants of EN.

\section{Author Contributions:}

Mahshid Sadat Ansari: acquisition of data, or analysis and interpretation of data.

Maryam Nasimi: drafting the manuscript or revising it critically for important intellectual content.

Kambiz Kamyab: acquisition of data, or analysis and interpretation of data.

Hamidreza Mahmoudi: conception and design, Given final approval of the version to be published.

\section{References}

1. Adams BB, Mutasim DF. Adult onset verrucous epidermal nevus. Journal of the American Academy of Dermatology. 1999;41(5):824-6.

2. Carbotti M, Coppola R, Graziano A, Verona Rinati M, Paolilli FL, Zanframundo S, et al. Dermoscopy of verrucous epidermal nevus: large brown circles as a novel feature for diagnosis. International journal of dermatology. 2016;55(6):653-6.

3. Sadeghinia A, Ghanadan A, Ehsani A, Noormohammadpour P, Ansari MS. Can dermoscopy open a new way to diagnosing Merkel cell carcinoma?. International journal of dermatology. 2019 Mar;58(3):e68-71. 
4. Ramam M. Linear epidermolytic acanthoma or adult-onset verrucous epidermal nevus? Indian journal of dermatology, venereology and leprology. 2010;76(5):563.

5. Martínez VA, Villarreal GEC, Ramos Garibay A, Mondragón MER. Verrucose epidermal nevus with belated grow and pregnancy. Case report. Ginecología y Obstetricia de México. 2007;75(10):636-40.

6. Su W. Histopathologic varieties of epidermal nevus. A study of 160 cases. The American Journal of Dermatopathology. 1982;4(2):161-70.

7. Ross R, DiGiovanna JJ, Capaldi L, Argenyi Z, Fleckman P, Robinson-Bostom L. Histopathologic characterization of epidermolytic hyperkeratosis: a systematic review of histology from the National Registry for Ichthyosis and Related Skin Disorders. Journal of the American Academy of Dermatology. 2008;59(1):86-90.

8. Verzi AE, Lacarrubba F, Quattrocchi E, Micali G. Verrucous Epidermal Nevus: Dermoscopy, Reflectance Confocal Microscopy, and Histopathological Correlation. Dermatol Pract Concept. 2019;9(3):230-1.

9. Gomes RT, Vargas PA, Tomimori J, Lopes MA, Santos-Silva AR. Linear verrucous epidermal nevus with oral manifestations: report of two cases. Dermatol Online J. 2020 Jan 15;26(1). pii: 13030/qt46n048wn.

10. Elmas ÖF. Dermoscopic aspect of verrucous epidermal nevi: New findings. Turk J Med Sci. 2019 Jun 18;49(3):710-714. doi: 10.3906/sag-1811-27.

11. Arjona-Aguilera C, Collantes-Rodriguez C, Villegas-Romero I, Ossorio-Garcia L, Jimenez-Gallo D, Linares-Barrios M. Rounded and velvety epidermal naevus: Dermoscopic findings and literature review. Australasian Journal of Dermatology. 2018 May;59(2):e166-7.

Table 1.Dermoscopic features in previous studies

\begin{tabular}{llllll}
\hline & & Carbotti et al. ${ }^{2}(\mathrm{~N}=8)$ & Verzi et al. $^{8}(\mathrm{~N}=9)$ & Elmas et al. ${ }^{10}(\mathrm{~N}=20)$ & $\mathrm{Ar}$ \\
\hline Sex & Sex & $\mathrm{F}=6 / \mathrm{M}=2$ & - & $\mathrm{F}=9 / \mathrm{M}=11$ & $\mathrm{M}$ \\
Lecatian age(year) & Median age(year) & 38 & - & 24 & 10 \\
& Trunk & $5 / 8$ & - & $7 / 20$ & - \\
& face & $1 / 8$ & - & $9 / 20$ & - \\
& Neck & $2 / 8$ & - & $4 / 20$ & - \\
Histopathology & Extremity & Histopathology & VEN & - & Ac \\
Dermoscopic findings & Large brown circles & $8 / 8$ & - & VEN & - \\
& Comedo-like opening & $3 / 8$ & $9 / 9$ & $10 / 20$ & - \\
& Cerebriform pattern & - & - & $7 / 20$ & + \\
& Thick brown line & - & - & $1 / 20$ & - \\
& globule & - & - & $9 / 20$ & $+(1)$ \\
& Small brown circles & - & - & $-(\mathrm{brown})$ & - \\
& Dotted vessels & - & - & - & - \\
\hline
\end{tabular}

+ Verrucous epidermal nevus is defined by hyperkeratosis, papillomatosis, acanthosis and elongation of rete ridges.

++ Other more frequent findings were Whitish and brown exophytic papillary structures, terminal hair and scale

Figure legends:

Figure 1. (a) Unilateral brown papules along blaschko's lines. (b, c) Dermoscopic examination: large and small brown circles in pigmented background

Figure 2. (a) Section shows epidermis with elongated rete ridge and orthokeratotic hyperkeratosis (H\&E, 
magnification $\times 10$ ). (b) High magnification reveals vacuolated cells. $(H \& E$, magnification $\times 40)$

\section{Hosted file}

title.docx available at https://authorea.com/users/319954/articles/449607-adult-onsetepidermal-nevus-with-epidermolytic-hyperkeratotic-pattern-case-report-dermoscopicfeatures-and-review-of-the-literature

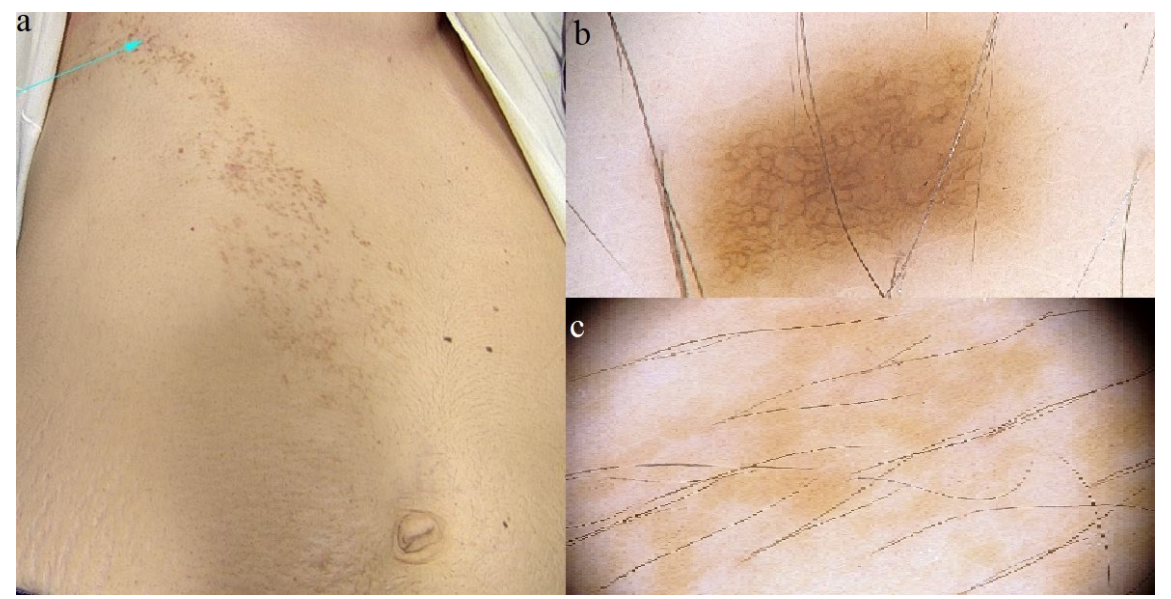




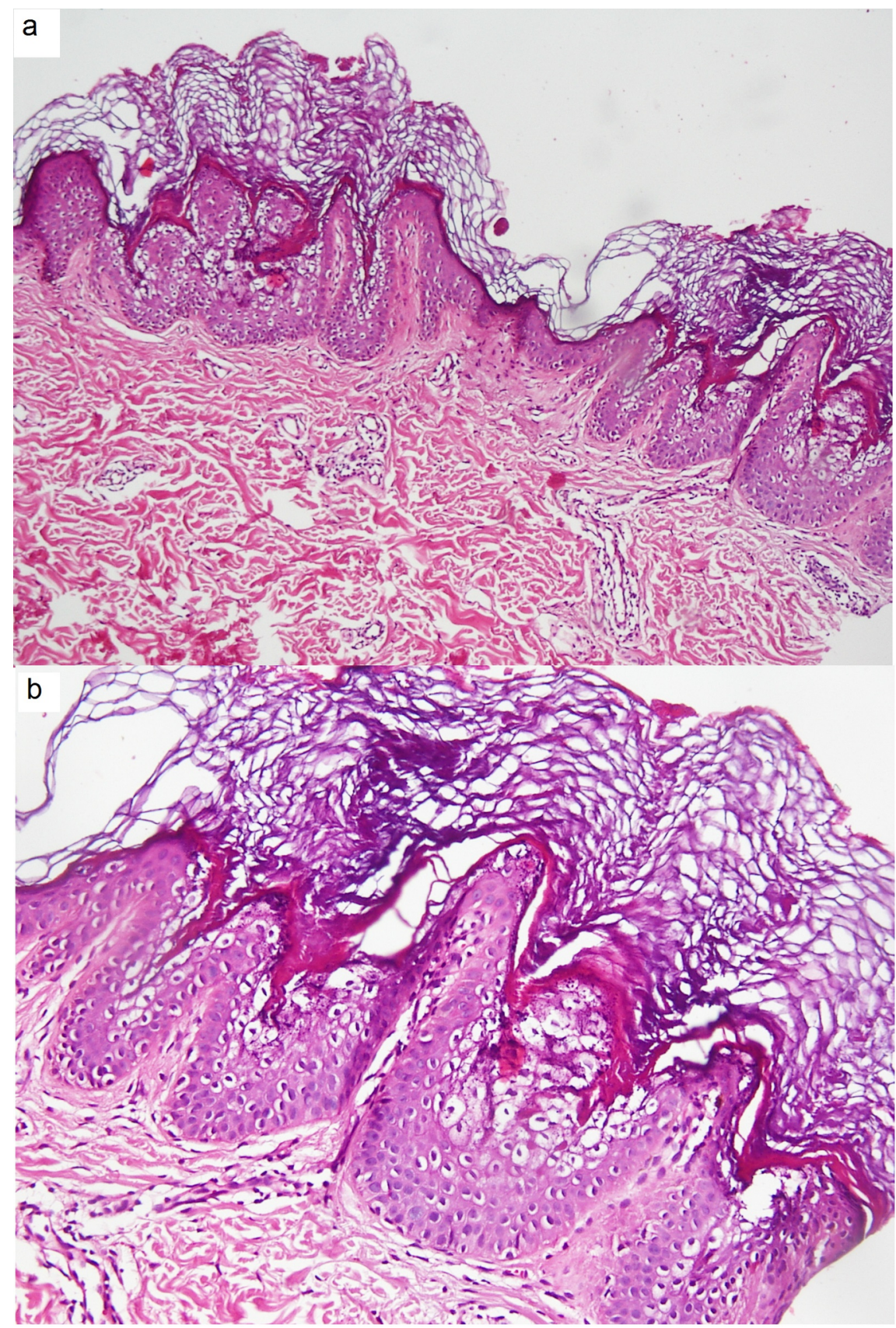

